\title{
Vitamin D supplements: a novel therapeutic approach for Alzheimer patients
}

\author{
Cedric Annweiler ${ }^{1,2}{ }^{*}$, Spyridon N. Karras ${ }^{3}$, Panagiotis Anagnostis ${ }^{3}$ and Olivier Beauchet ${ }^{1}$ \\ ' Department of Geriatric Medicine, UPRES EA 4638, University Hospital, Angers, France \\ ${ }^{2}$ Robarts Research Institute, University of Western Ontario, London, ON, Canada \\ ${ }^{3}$ Unit of Reproductive Endocrinology, First Department of Obstetrics and Gynecology, Medical School, Aristotle University of Thessaloniki, Thessaloniki, Greece \\ *Correspondence: ceannweiler@chu-angers.fr \\ Edited by: \\ Cesare Mancuso, Catholic University School of Medicine, Italy \\ Reviewed by: \\ Robert Nistico, University of Calabria, Italy
}

Keywords: vitamin D, neuroendocrinology, Alzheimer disease, dementia, research, multi-target drug, disease-modifying drug, memantine

\section{INTRODUCTION}

Alzheimer disease $(\mathrm{AD})$ is the main cause of dementia and loss of functional independence in the elderly (Aisen, 2010; Vellas and Aisen, 2010). AD is a chronic neurodegenerative disease characterized by a progressive decline of cognitive performance with a deleterious impact on social activities. $\mathrm{AD}$ is a worldwide big concern because of its adverse consequences and its expanding prevalence and incidence (Aisen, 2010; Vellas and Aisen, 2010). In order to reduce $\mathrm{AD}$ impact at an individual level and in terms of health and social costs, the development of efficient therapeutic strategies proves necessary. Regrettably, the development of all current specific medications is unsuccessful in clinical development phases (Birks, 2006; McShane et al., 2006; Anand et al., 2014). However, an interesting fact is that the prevalence of dementia in the general population might still be subject to changes. Indeed, a reduction in the prevalence of dementia is reported among English community-dwellers over the past two decades (Matthews et al., 2013). So, even if some factors, such as the increased life expectancy beyond 80 years, are unavoidable and augment the global prevalence of dementia, others may instead reduce its prevalence. For instance, efficient primary prevention of cardio-vascular disease is suspected to explain the reduced prevalence of dementia in England (Matthews et al., 2013). In the same vein, vitamin D, an "old" molecule primarily known for its effects on the bone, has been singled out in the last decade as an important biological component able to influence the natural history of AD. Here, we wish to highlight the difficulties experienced by the research on curative treatments and, in contrast, the prospects offered by multitarget drugs, especially those based on vitamin D.

\section{TREATMENTS RESEARCH IN ALZHEIMER DISEASE: THE IRONY OF CURATIVE THERAPIES}

The only drugs currently available (i.e., cholinesterase inhibitors and memantine) slow down without changing the natural history of AD (Birks, 2006; McShane et al., 2006). Because of their symptomatic action, they are intended only for patients with mild-to-severe AD. Thus, one of the main $\mathrm{AD}$ challenges over the coming decade lies in the finding of a curative drug (Vellas and Aisen, 2010). Recent research has focused on "diseasemodifying" medications to intervene in the pathogenesis of the disease, able to halt or slow the neurodegenerative process. To date, strategies to develop diseasemodifying drugs have mainly targeted amyloid- $\beta$ peptide (accumulation, aggregation, clearance) and/or the "tubulin associated unit" (TAU protein: phosphorylation and aggregation) by active and passive immunotherapy (Vellas and Aisen, 2010; Anand et al., 2014). Unfortunately candidate drugs, such as bapineuzumab, have failed in phase III clinical trials conducted in mild-to-moderate AD (Anand et al., 2014).

Because the earlier the diseasemodifying treatment starts, the greater is the possibility of efficacy, it is important to initiate the treatment before the clinical manifestation of the overt disease. This is one of the reasons of the interest for the early diagnosis of $\mathrm{AD}$. Importantly, this research has identified new morphological as well as functional and biological criteria that, combined with the classical neuropsychological expertise, help make the diagnosis of $\mathrm{AD}$ earlier and earlier (Schrötter et al., 2013). Such accuracy of the diagnosis of early-stage $\mathrm{AD}$ led in memory clinics to a drastic selection of participants for the curative clinical trials, with consequent substantial recruitment difficulties. The second consequence of this hyperselection was to address only a fraction of the patients followed in memory clinics, predominantly those with the prodromal stage of AD. For instance, the French National Centre for the Management of Trials on Healthcare Products (CeNGEPS), which involves more than ten university memory clinics (UMCs) in France, included in 2009 only 260 participants in clinical trials, although there were potentially 24,000 patients available in these centers. In other words, the curative drugs are addressing only $1.1 \%$ of $\mathrm{AD}$ patients followed in French UMCs. The question is then what can be offered to all other patients.

\section{TREATMENTS RESEARCH IN ALZHEIMER DISEASE: THE MULTI-TARGET DRUGS}

In addition to curative treatments, two other therapeutic approaches should be considered in AD. The first one is the nondrug-based approach, which is a multidomain intervention designed to stimulate 
AD patients (de Sant'Anna and Morat, 2013). The second one is the multitarget drug approach, meaning that the treatment administered to $\mathrm{AD}$ patients simultaneously targets several neurodegenerative processes (Cavalli et al., 2008). The latter approach offers the opportunity to combine current standard antidementia symptomatic drugs with other neuroprotective agents to build a multiregimen with multi-target effects. This questioning is central since patients at later stages of $\mathrm{AD}$ (i.e., with symptoms) could benefit from this new therapeutics, thus reducing chance inequalities between $\mathrm{AD}$ patients. In addition, it could benefit a larger number of patients and could be easily implemented compared with current trials, since it targets diagnosed patients who are already using a symptomatic treatment. Among the candidate molecules is the vitamin $\mathrm{D}$, which has demonstrated in the last decade numerous neurological effects.

\section{VITAMIN D AND ALZHEIMER DISEASE: FROM AN INTRIGUING IDEA TO A THERAPEUTIC OPTION}

About one billion people are deficient in vitamin $\mathrm{D}$ throughout the world. Older adults are especially affected, in particular those with $\mathrm{AD}$ with a prevalence of approximately 70-90\% (Holick, 2007; Annweiler et al., 2011).

Besides its classical function of bone metabolism regulation, vitamin $\mathrm{D}$ has exhibited multiple biological targets mediated by its nuclear hormone receptor, the Vitamin D Receptor (VDR) (Kalueff and Tuohimaa, 2007; Annweiler et al., 2010). Specific actions on target organs such as the central nervous system (CNS) have been described, providing evidence for a neurosteroid action of vitamin $D$ (Kalueff and Tuohimaa, 2007). In particular, VDRs are present in neurons and glial cells of the hippocampus, cortex and sub-cortex, all regions essential to cognition. The binding of vitamin $\mathrm{D}$ on the VDRs triggers neuronal protection against $\mathrm{AD}$ degenerative processes, including anti-inflammatory action, antioxidant effect, control of calcium homeostasis by regulating the concentration of intracellular calcium in hippocampal neurons, anti-atrophic effect by regulating neurotrophic agents, attenuation of $\mathrm{A} 342$ peptide accumulation by stimulating the phagocytosis of $A ß$ peptide together with enhancing brain-to-blood $A ß$ efflux transport at the blood-brain barrier, and the prevention of acetylcholine defect by increasing the activity of choline acetyltransferase (thus the bioavailability of acetylcholine) in the brain (Kalueff and Tuohimaa, 2007; Annweiler et al., 2010; Annweiler and Beauchet, 2011). These experimentally-described neuroprotective properties of vitamin D may help, in the case of normalized vitamin D status, to address against the decline of brain function in $\mathrm{AD}$, especially against cognitive decline (Figure 1).

Epidemiology is consistent with this notion, and has repeatedly shown that low serum concentrations of vitamin D are cross-sectionally associated with global cognitive impairment (Annweiler et al., 2009), the people with hypovitaminosis $\mathrm{D}$ having more cognitive disorders (Etgen et al., 2012), specifically more executive dysfunctions (Annweiler et al., 2013a). Specifically, it has been reported that people with $\mathrm{AD}$ have lower vitamin $\mathrm{D}$ status than controls (Annweiler et al., 2013b). They also exhibit increased levels of vitamin D-binding protein (Moon et al., 2013) and lower levels of 25hydroxyvitamin D in the cerebrospinal fluid (Johansson et al., 2013) compared to controls. Longitudinal prospective studies have enabled to better understand this association and have reported a temporal sequence, with hypovitaminosis $\mathrm{D}$ preceding the onset of cognitive decline (Llewellyn et al., 2010).

Recent biomedical literature further suggests that the VDRs may confer genetic risk for AD. Some human variants appear less sensitive to vitamin $\mathrm{D}$ and more likely to experience cognitive decline. For instance, $\mathrm{AD}$ patients have reduced levels of VDR mRNA (Sutherland et al., 1992). In contrast, it has been reported that the overexpression of VDR may suppress amyloid precursor protein transcription (Wang et al., 2012). A significant association has also been shown between the VDR gene APA1 polymorphism and the onset of $\mathrm{AD}$, the $A a$ genotype multiplying by 2.3 the risk of $\mathrm{AD}$ compared to the $A A$ genotype (Gezen-Ak et al., 2007).

It seems thus crucial to maintain high vitamin D levels in the elderly, especially in those with $\mathrm{AD}$. In line with this, high intake of vitamin D (whether from food, supplements, or sun exposure) is associated with better cognitive function in older individuals. Having more than 800UI of vitamin $\mathrm{D}$ supplies per day divides the risk of $\mathrm{AD}$ by 5 after 7 years of followup (Annweiler et al., 2012a). This neuroprotective effect has been confirmed by clinical comparative trials reporting cognitive improvement after vitamin D supplementation whether in general aged population (Prybelski et al., 2008; Annweiler et al., 2012b) or in patients who already have symptoms of AD (Stein et al., 2011). The cognitive benefits of supplementation appear at 4 weeks (Prybelski et al., 2008), and particularly for executive functions and processing speed (Annweiler et al., 2013a).

It has to be noted yet that, although most of older adults have hypovitaminosis D, not all develop AD. It is thus unlikely that hypovitaminosis $\mathrm{D}$ alone explains the onset of $\mathrm{AD}$, and that vitamin D supplementation is sufficient to prevent $\mathrm{AD}$ by itself. One solution might thus be to combine vitamin $\mathrm{D}$ with a symptomatic anti-dementia treatment to build a multi-target drug (Annweiler and Beauchet, 2012). Indeed, if hypovitaminosis D explains in part the pathological process of $\mathrm{AD}$, it may also enhance the effectiveness of standard anti-dementia treatments or account at least partially for the resistance to these treatments. Even speculative, this engages clinicians to replenish vitamin D before starting anti-dementia treatments or to use vitamin $\mathrm{D}$ as an adjunct to standard treatments. In line with this, a recent 6-month controlled trial has recently reported that the combination of memantine + vitamin $\mathrm{D}$ was superior to memantine alone and vitamin $\mathrm{D}$ alone in preventing cognitive decline among $\mathrm{AD}$ participants (Annweiler et al., 2012c). In fact, those taking both molecules had a clinically relevant and statistically significant gain of 4 points on the Mini-Mental State Examination. These results were consistent with an in vitro experiment, which showed that cortical axons degenerate less after exposure to amyloid- $\beta$ peptide or glutamate in microfluidic neuronal cultures enriched with memantine plus vitamin D compared to control medium and compared 


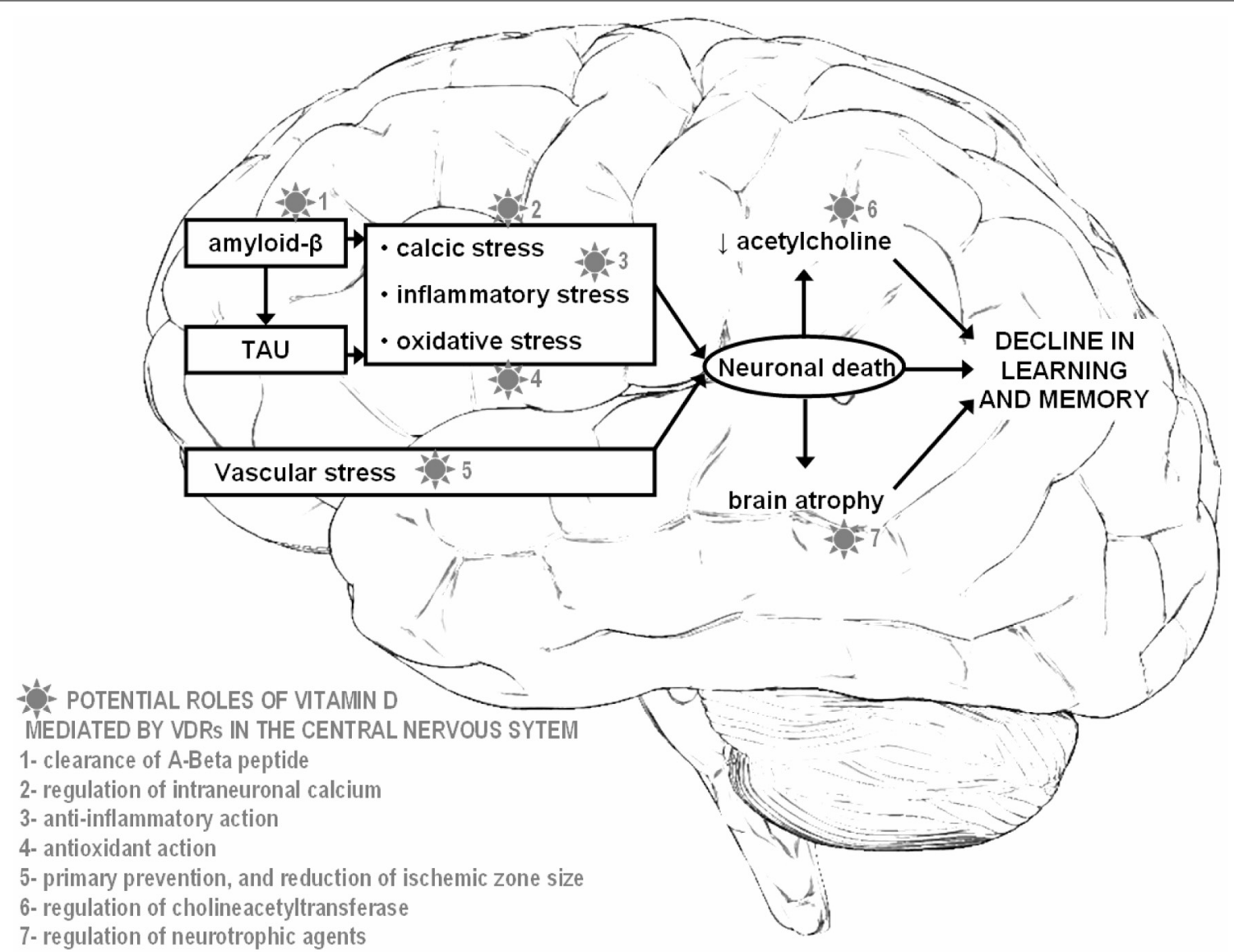

FIGURE 1 | Potential neuroprotective roles offered by vitamin D in Alzheimer disease.

to cultures enriched with memantine only or with vitamin D only (Annweiler et al., 2014).

\section{CONCLUSIONS}

In conclusion, $\mathrm{AD}$ is a public health concern due to its very high prevalence and because the few drugs currently available are only symptomatic. Current therapeutic research, which focuses mainly on curative disease-modifying treatments, addresses a very small fraction of $\mathrm{AD}$ patients, predominantly those with prodromal disease and no symptoms of dementia. It is thus necessary to consider the patients who already suffer from moderate-to-severe $\mathrm{AD}$, who constitute the majority of $\mathrm{AD}$ patients. One of the best hopes for them may rely on the development of multitarget drugs. In particular, using vitamin D supplements as an adjunct to standard anti-dementia treatments appears to be a simple, inexpensive, and efficient therapeutic strategy and leads to encouraging prospects for the correction of neurological disorders in $\mathrm{AD}$.

\section{AUTHORS CONTRIBUTIONS}

All authors meet all of the following criteria: (1) Contributing to the conception and design, or analyzing and interpreting data; (2) Drafting the article or revising it critically for important intellectual content; and (3) Approving the final version to be published.

\section{REFERENCES}

Aisen, P. S. (2010). Pre-dementia Alzheimer's trials: overview. J. Nutr. Health Aging 14, 294. doi: 10.1007/s12603-010-0065-2

Anand, R., Gill, K. D., and Mahdi, A. A. (2014). Therapeutics of Alzheimer's disease: past, present and future. Neuropharmacology 76, 27-50. doi: 10.1016/j.neuropharm.2013.07.004

Annweiler, C., Allali, G., Allain, P., Bridenbaugh, S., Schott, A. M., Kressig, R. W., et al. (2009). Vitamin $\mathrm{D}$ and cognitive performance in adults: a systematic review. Eur. J. Neurol. 16, 1083-1089. doi: 10.1111/j.1468-1331.2009.02755.x
Annweiler, C., and Beauchet, O. (2011). Vitamin Dmentia: randomized clinical trials should be the next step. Neuroepidemiology 37, 249-258. doi: 10.1159/000334177

Annweiler, C., and Beauchet, O. (2012). Possibility of a new anti-alzheimer's disease pharmaceutical composition combining memantine and vitamin D. Drugs Aging 29, 81-91. doi: 10.2165/11597550000000000-00000

Annweiler, C., Brugg, B., Peyrin, J. M., Bartha, R., and Beauchet, O. (2014). Combination of memantine and vitamin $\mathrm{D}$ prevents axon degeneration induced by amyloid-beta and glutamate. Neurobiol. Aging 35, 331-335. doi: 10.1016/j.neurobiolaging.2013.07.029

Annweiler, C., Montero-Odasso, M., Llewellyn, D. J., Richard-Devantoy, S., Duque, G., and Beauchet, O. (2013a). Meta-analysis of memory and executive dysfunctions in relation to vitamin D. J. Alzheimers Dis. 37, 147-171. doi: 10.3233/JAD130452

Annweiler, C., Llewellyn, D. J., and Beauchet, O. (2013b). Low serum vitamin D concentrations in Alzheimer's disease: a systematic review and meta-analysis. J. Alzheimers Dis. 33, 659-674. doi: 10.3233/JAD-2012-121432

Annweiler, C., Rolland, Y., Schott, A. M., Blain, H., Vellas, B., Herrmann, F. R., et al. (2012a). Higher 
vitamin D dietary intake is associated with lower risk of Alzheimer's disease: a 7-year follow-up. J. Gerontol. A Biol. Sci. Med. Sci. 67, 1205-1211. doi: 10.1093/gerona/gls107

Annweiler, C., Fantino, B., Gautier, J., Beaudenon, M., Thiery, S., and Beauchet, O. (2012b). Cognitive effects of vitamin D supplementation in older outpatients visiting a memory clinic: a pre-post study. J. Am. Geriatr. Soc. 60, 793-795. doi: 10.1111/j.1532-5415.2011.03877.x

Annweiler, C., Herrmann, F. R., Fantino, B., Brugg, B., and Beauchet, O. (2012c). Effectiveness of the combination of memantine plus vitamin D on cognition in patients with Alzheimer disease: a prepost pilot study. Cogn. Behav. Neurol. 25, 121-127. doi: 10.1097/WNN.0b013e31826df647

Annweiler, C., Schott, A. M., Berrut, G., Chauviré, V., Le Gall, D., Inzitari, M., et al. (2010). Vitamin D and ageing: neurological issues. Neuropsychobiology 62, 139-150. doi: 10.1159/000318570

Annweiler, C., Souberbielle, J. C., Schott, A. M., de Decker, L., Berrut, G., and Beauchet, O. (2011). Vitamin D in the elderly: 5 points to remember. Geriatr. Psychol. Neuropsychiatr. Vieil. 9, 259-267. doi: 10.1684/pnv.2011.0288

Birks, J. (2006). Cholinesterase inhibitors for Alzheimer's disease. Cochrane Database Syst. Rev. CD005593. doi: 10.1002/14651858.CD005593

Cavalli, A., Bolognesi, M. L., Minarini, A., Rosini, M., Tumiatti, V., Recanatini, M., et al. (2008). Multi-target-directed ligands to combat neurodegenerative diseases. J. Med. Chem. 51, 347-372. doi: $10.1021 /$ jm7009364

de Sant'Anna, M., and Morat, B. (2013). Non-drugbased management of Alzheimer's disease. Soins Gerontol. 102, 15-18.

Etgen, T., Sander, D., Bickel, H., Sander, K., and Förstl, H. (2012). Vitamin D deficiency, cognitive impairment and dementia: a systematic review and meta-analysis. Dement. Geriatr. Cogn. Disord. 33, 297-305. doi: 10.1159/000339702

Gezen-Ak, D., Dursun, E., Ertan, T., Hanagasi, H., Gürvit, H., Emre, M., et al. (2007). Association between vitamin D receptor gene polymorphism and Alzheimer's disease. Tohoku J. Exp. Med. 212, 275-282. doi: 10.1620/tjem.212.275

Holick, M. F. (2007). Vitamin D deficiency. N. Engl. J. Med. 357, 266-281. doi: 10.1056/ NEJMra070553
Johansson, P., Almqvist, E. G., Johansson, J. O., Mattsson, N., Andreasson, U., Hansson, O. et al. (2013). Cerebrospinal fluid (CSF) 25hydroxyvitamin D concentration and CSF acetylcholinesterase activity are reduced in patients with Alzheimer's disease. PLOS ONE 8:e81989. doi: 10.1371/journal.pone.0081989

Kalueff, A. V., and Tuohimaa, P. (2007). Neurosteroid hormone vitamin D and its utility in clinical nutrition. Curr. Opin. Clin. Nutr. Metab. Care 10, 12-19. doi: 10.1097/MCO.0b013e328010ca18

Llewellyn, D. J., Lang, I. A., Langa, K. M., MunizTerrera, G., Phillips, C. L., Cherubini, A., et al. (2010). Vitamin D and risk of cognitive decline in elderly persons. Arch. Intern. Med. 170, 1135-1141. doi: 10.1001/archinternmed.2010.173

Matthews, F. E., Arthur, A., Barnes, L. E., Bond, J., Jagger, C., Robinson, L., et al. (2013). Medical Research Council Cognitive Function and Ageing Collaboration. A two-decade comparison of prevalence of dementia in individuals aged 65 years and older from three geographical areas of England: results of the Cognitive Function and Ageing Study I and II. Lancet 382, 1405-1412. doi: 10.1016/S0140-6736(13) 61570-6

McShane, R., Areosa Sastre, A., and Minakaran, N. (2006). Memantine for dementia. Cochrane Database Syst. Rev. CD003154. doi: 10.1002/14651858.CD003154.pub5

Moon, M., Song, H., Hong, H. J., Nam, D. W., Cha, M. Y., Oh, M. S., et al. (2013). Vitamin D-binding protein interacts with Abeta and suppresses Abeta-mediated pathology. Cell Death Differ. 20, 630-638. doi: 10.1038/cdd.2012.161

Prybelski, R., Agrawal, S., Krueger, D., Engelke, J. A., Walbrun, F., and Binkley, N. (2008). Rapid correction of low vitamin D status in nursing home residents. Osteoporos. Int. 19, 1621-1628. doi: 10.1007/s00198-008-0619-x

Schrötter, A., Magraoui, F. E., Gröttrup, B., Wiltfang, J., Heinsen, H., Marcus, K., et al. (2013). Early diagnosis of neurodegenerative diseases-the long awaited Holy Grail and bottleneck of modern brain research-19th HUPO BPP workshop: May 22-24, 2013, Dortmund, Germany. Proteomics 13, 2938-2941. doi: 10.1002/pmic.201370164

Stein, M. S., Scherer, S. C., Ladd, K. S., and Harrison, L. C. (2011). A randomized controlled trial of high-dose vitamin D2 followed by intranasal insulin in Alzheimer's disease. J. Alzheimers Dis. 26, 477-484. doi: 10.3233/JAD-2011110149

Sutherland, M. K., Somerville, M. J., Yoong, L. K. Bergeron, C., Haussler, M. R., and McLachlan, D. R. (1992). Reduction of vitamin D hormone receptor mRNA levels in Alzheimer as compared to Huntington hippocampus: correlation with calbindin-28k mRNA levels. Brain Res. Mol. Brain Res. 13, 239-250. doi: 10.1016/0169328X(92)90032-7

Vellas, B., and Aisen, P. S. (2010). Early Alzheimer's trials: new developments. J. Nutr. Health Aging 14, 293. doi: 10.1007/s12603-0100064-3

Wang, L., Hara, K., Van Baaren, J. M., et al. (2012). Vitamin D receptor and Alzheimer's disease: a genetic and functional study. Neurobiol. Aging 33, 1844.e1-1844.e9. doi: 10.1016/j.neurobiolaging.2011.12.038

Conflict of Interest Statement: The concept of combining memantine with vitamin $\mathrm{D}$ in the prevention and treatment of Alzheimer's disease and related disorders was patented by Angers University Hospital and the University of Angers. The authors report no conflicts of interest with this research. None of the authors have a personal financial interest in this research.

Received: 09 December 2013; accepted: 08 January 2014; published online: 28 January 2014

Citation: Annweiler C, Karras SN, Anagnostis $P$ and Beauchet $O$ (2014) Vitamin D supplements: a novel therapeutic approach for Alzheimer patients. Front. Pharmacol. 5:6. doi: 10.3389/fphar.2014.00006 This article was submitted to Experimental Pharmacology and Drug Discovery, a section of the journal Frontiers in Pharmacology.

Copyright (c) 2014 Annweiler, Karras, Anagnostis and Beauchet. This is an open-access article distributed under the terms of the Creative Commons Attribution License (CC BY). The use, distribution or reproduction in other forums is permitted, provided the original author(s) or licensor are credited and that the original publication in this journal is cited, in accordance with accepted academic practice. No use, distribution or reproduction is permitted which does not comply with these terms. 